\title{
Paediatric Enterobacteriaceae infections in hospitalised children in Durban, KwaZulu-Natal
}

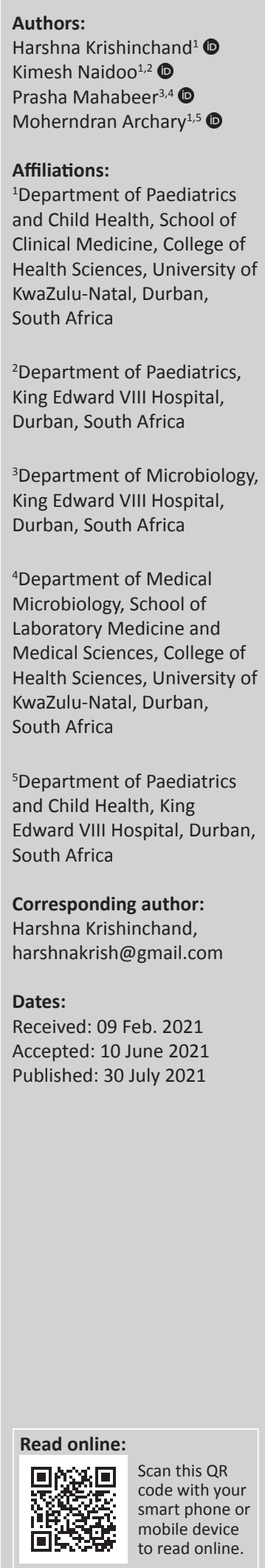

Background: Community-acquired Gram-negative Enterobacteriaceae infections in malnourished and HIV-infected hospitalised children are not well documented and are of concern because of increasing antibiotic resistance and limited available treatment options. This study describes the clinical characteristics and outcomes of hospitalised children with positive Enterobacteriaceae cultures.

Method: A retrospective chart review of children with Gram-negative Enterobacteriaceae infections was performed in King Edward VIII Hospital, a referral hospital in Durban, KwaZulu-Natal. Standard descriptive and analytical statistics, including regression analysis, were performed to determine the clinical characteristics associated with Enterobacteriaceae infections in children hospitalised in the study period.

Results : Of all hospitalised children in the study period, 207 (3.5\%) had positive cultures for Enterobacteriaceae isolates, with Escherichia coli 109 (44.5\%) and Klebsiella spp. 59 (24.1\%) making up most of the infections. Urine $(126 ; 58 \%)$ followed by stool $(34 ; 14.8 \%)$ and blood (35; 14.0\%) were the commonest samples that yielded positive cultures. Diarrhoeal hospitalisations especially posed a higher risk for Enterobacteriaceae infections. Severe acutely malnourished and HIV-infected children were at higher risk. These comorbidities were independently associated with an increased risk of Enterobacteriaceae infection. Prolonged hospitalisation and increased risk of death were also associated with Enterobacteriaceae infection.

Conclusion: Enterobacteriaceae infections were common in hospitalised children and posed an increased risk, especially in malnourished and HIV-infected children. Further studies investigating the relationships between diarrhoea, urinary tract infections and Enterobacteriaceae infections are needed.

Keywords: paediatric; Enterobacteriaceae infections; HIV; malnutrition; hospitalisation.

\section{Introduction}

Sub-Saharan Africa continues to have high under-five mortality rates fuelled by the burdens of malnutrition and multiple childhood infections. ${ }^{1,2,3}$ Gram-negative Enterobacteriaceae infections are of particular concern because of an increasing incidence and the emergence of broad-spectrum antibiotic resistance, limiting the available treatment options. ${ }^{4,5}$ The extent of Gram-negative Enterobacteriaceae infections has been well documented in adults, but this is not the case in children, especially in populations with high HIV and malnutrition rates. ${ }^{6,7}$

Severely malnourished children have a threefold higher case fatality than children with normal nutritional status. ${ }^{8}$ Although the current standard management guidelines for children hospitalised with severe acute malnutrition (SAM) recommend empiric antibiotics (ampicillin and gentamicin or ceftriaxone for severe illness), these antibiotics may be less effective in treating Enterobacteriaceae infections., ${ }^{910}$ Understanding the incidence and antimicrobial resistance patterns of Gram-negative isolates from malnourished children will better inform antibiotic usage in malnourished children and will influence the choice of antibiotics in this vulnerable population.

Invasive bacterial enteric pathogens, including Shigella, Campylobacter and Enteroaggregativeadherent E. coli (EAEC), have been associated with gut inflammation and barrier disruption triggering chronic diarrhoea that can lead to malabsorption and malnutrition. ${ }^{11}$

How to cite this article: Krishinchand $\mathrm{H}$, Naidoo K, Mahabeer $\mathrm{P}$, Archary M. Paediatric Enterobacteriaceae infections in hospitalised children in Durban, KwaZulu-Natal. S Afr J Infect Dis. 2021;36(1), a279. https://doi.org/10.4102/sajid.v36i1.279

Copyright: (C 2021. The Authors. Licensee: AOSIS. This work is licensed under the Creative Commons Attribution License. 
Multiple factors such as organ or stem cell transplant, intensive care unit admission, prolonged hospitalisation, surgery and care in long-term care facilities have been highlighted as risk factors for infection with drug-resistant Enterobacteriaceae in adults. ${ }^{12}$

In a review summarising six studies of carbapenem-resistant Enterobacteriaceae infections, 64 isolates were cultured from 63 children. The median age of infected children was 1 year (0-17 years), males (67\%) were affected more than females. Many children (53\%) were hospitalised to an intencive care unit (ICU) and probably had hospital-acquired infections. A total of $87 \%$ had been hospitalised for more than $48 \mathrm{~h}$. Comorbidities identified included pulmonary disease, prematurity, leukaemia or solid tumours, cardiac disease, necrotising enterocolitis, solid organ or stem cell receipt. ${ }^{13}$

Extended-spectrum $\beta$-lactamase (ESBL) producing Enterobacteriaceae have emerged as a major cause of healthcareassociated infections with significant morbidity and mortality in children. ${ }^{12,13}$.

In populations with high HIV endemicity, children are hospitalised with poor nutritional status and opportunistic infections, including tuberculosis. The relationship to Enterobacteriaceae infection has not been completely described and explained.

There are limited therapeutic options available for multidrug resistant Gram-negative bacterial infections in children. ${ }^{7}$ Ideal treatment regimens are hampered by a scarcity of randomised trials assessing the efficacy of various drug options in children with community-acquired Enterobacteriaceae infections. ${ }^{14,15}$. Morbidity and mortality associated with these infections are high. Reports on epidemiology and risk factors for infection in children are limited. 7,12,13,15.

This study aimed to describe the clinical characteristics of children who were hospitalised at King Edward VIII Hospital $(\mathrm{KEH})$, Durban, from whom an Enterobacteriaceae was cultured.

\section{Methodology}

A retrospective, descriptive, observational study of all children hospitalised at $\mathrm{KEH}$, who cultured an Enterobacteriaceae from any site, was conducted from the period January 2016 to 31 December 2017. All hospitalised children are admitted to an acute admission ward under the care of a specialist paediatrician. Decisions on specimen collection for specific microbiological cultures were made at the discretion of the treating clinician.

The National Health Laboratory Service (NHLS) was used to access all positive Enterobacteriaceae cultures, from any site, in children between the ages of $>28$ days and $<13$ years for the study period. This study excluded neonates (who have a higher Gram-negative burden of infections) and reflects a general hospitalised paediatric population with an expected lower burden of such infections.
The patient files of all children who had positive Enterobacteriaceae cultures were sourced from the $\mathrm{KEH}$ archives, and the following data were extracted: age, gender, final clinical diagnosis, HIV status, nutritional status, clinical outcome, mortality and length of stay (LOS). Anthropometric measurements from the patient files were used to classify nutritional status using World Health Organisation $(\mathrm{WHO})$ growth standards. Patients were classified: no malnutrition (NAM), moderate acute malnutrition (MAM), severe acute malnutrition (SAM) (with or without oedema) and overweight for age (OWFA) ${ }^{16}$.

The HIV status of infants was determined from the last verified HIV test carried out within the health system and results were also verified on the NHLS information system. They were categorised as: HIV-infected, HIV-uninfected, HIV-exposed uninfected and HIV-exposed unknown. For the exposed group without confirmation of HIV results, these patients were categorised as unknown (no confirmation of HIV results). Patients infected with HIV were further categorised as receiving antiretroviral treatment (ART) or not. Patients with an unknown HIV result (107/5946; 1.8\%) were excluded from the non-descriptive analysis.

Information of the sites of culture, types of organisms and antibiotic sensitivity were extracted and verified from the NHLS information system. All data were extracted into an excel spreadsheet.

Data from the study cohort was compared with routinely collected data on all children hospitalised to the paediatric medical wards at $\mathrm{KEH}$ during the study period.

\section{Data analysis}

Descriptive and inferential statistical analysis methods were used for understanding and deriving meaningful conclusions from the collected data. Summary measures such as the minimum, maximum, quartiles, interquartile range, mean, standard deviation and coefficient of variation were calculated for the descriptive statistics. The categorical variables were described as counts and percentage frequencies. To assess the mean difference of numerical variables across at least three levels of a categorical variable, the ANOVA test for normally distributed measurements or the Kruskal-Wallis test for the non-normally distributed measurements were used. To test the association between categorical variables, the Chi-square test or Fisher's exact test (small frequencies) were used. All the inferential statistical analysis tests were conducted at 5\% levels of significance.

\section{Ethical considerations}

Ethical approval was obtained from the University of KwaZulu-Natal (UKZN) Biomedical Research Ethics Committee (BREC Ref No: BE 125/19) and gatekeeper permission from the National Health Laboratory Service (NHLS) and King Edward VIII Hospital (KEH). 


\section{Results}

A total of 5946 children above 1 month were hospitalised to King Edward Hospital Paediatric Department during the study period 01 January 2016 and 31 December 2017. In this period, 207 of these hospitalised children (3.5\%) had positive Enterobacteriaceae cultures, of which $59.4 \%$ were community-acquired (defined as positive cultures less than $48 \mathrm{~h}$ after admission) and $40.6 \%$ were hospitalacquired defined as positive cultures more than $48 \mathrm{~h}$ after admission.

Figure 1 illustrates the most common organisms and the sites from which they were cultured. Escherichia coli (109; $44.5 \%)$ and Klebsiella spp. (59; 24.1\%) were the most commonly isolated organisms. The positive cultures were obtained from urine $(135 / 234 ; 57.7 \%)$, stool $(33 / 234 ; 14.1 \%)$, blood $(33 / 234 ; 14.1 \%)$, sputum $(21 / 234 ; 9 \%)$, skin swabs $(10 / 234 ; 4.3 \%)$, CSF $(1 ; 0.4 \%)$ and one site $(1 ; 0.4 \%)$, which was unknown $E$. coli was isolated predominately from urine and Klebsiella spp. from sputum. Extended-spectrum $\beta$-lactamase producing organisms were found in 51 patients, $33(65.0 \%)$ of whom were malnourished and $18(35.0 \%)$ HIV-infected. Only two of the 207 patients cultured extensively drug-resistant organisms (defined as nonsusceptibility to at least one agent in three or more antimicrobial categories). These were both Klebsiella spp. cultured from urine. Of the 59 isolates that were cultured ESBL producers, these were: Klebsiella spp. (27/59; 45.8\%), E. coli $(26 / 59 ; 44.1 \%)$ and the remaining $10 \%$ were Shigella $(2 / 59)$, both from stool samples and Morganella (1/59) cultured from sputum.

\section{Clinical characteristics of patients with Enterobacteriaceae infections}

Five patients in the study group were re-hospitalised within 8 weeks of discharge, and $13(6.3 \%)$ patients in the study group were transferred from other healthcare facilities. In some patients $(33 ; 15.9 \%)$, Enterobacteriaceae were cultured on more than one occasion during the same hospitalisation period. Seventeen samples were repeat samples.

In this public sector hospital, all paediatric hospitalisations are categorised into three main diagnostic groups: respiratory illness, diarrhoeal disease and 'other' (includes meningitis, urinary tract infection [UTI], septicaemia and others). Table 1 highlights that Enterobacteriaceae infections were significantly more common in children hospitalised with the diarrhoeal diagnosis. Table 1 compares the clinical characteristics of patients admitted with and without Enterobacteriaceae infections.

\section{Nutritional status}

Children hospitalised with SAM (with or without oedema) showed a significantly increased risk of Enterobacteriaceae infection (Table 2).

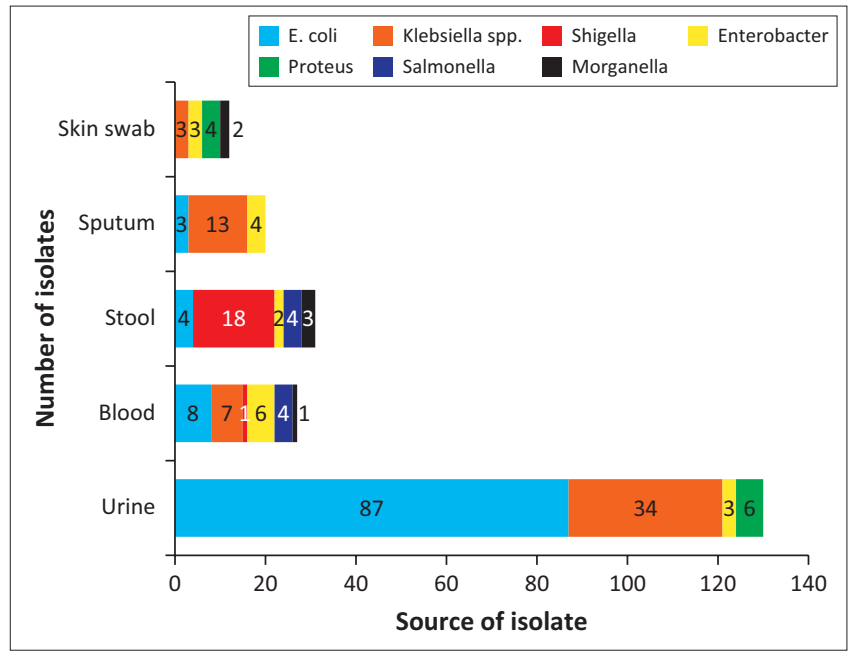

Note: The table includes only the main isolates and sites (Not included in the figure are Campylobacter (1 positive culture), 1 E. coli cultured in a site unspecified, 1 CSF cultured Salmonella and other Enterobacteriaceae species (11/234) including Citrobacter, Pantoea species and Serratia ficaria.

E.coli, Escherichia coli; spp., species.

FIGURE 1: Commonest Enterobacteriaceae isolates and sites cultured from.

TABLE 1: Age, gender, main diagnosis and Enterobacteriaceae infections.

\begin{tabular}{|c|c|c|c|c|c|}
\hline \multirow[t]{2}{*}{ Group } & \multicolumn{2}{|c|}{$\begin{array}{c}\text { No Enterobacteriaceae } \\
\text { infection }\end{array}$} & \multicolumn{2}{|c|}{$\begin{array}{c}\text { Enterobacteriaceae } \\
\text { infected }\end{array}$} & \multirow[t]{2}{*}{$p$} \\
\hline & $N=5739$ & $\%$ & $N=207$ & $\%$ & \\
\hline Gender & - & & - & & 0.078 \\
\hline Female & 2364 & 41.2 & 98 & 47.3 & - \\
\hline Male & 3373 & 58.8 & 109 & 52.7 & - \\
\hline Missing & 2 & 0.0 & 0 & 0 & - \\
\hline Age & - & & - & & 0.009 \\
\hline 28 days $-<1$ year & 2014 & 35.1 & 59 & 28.5 & 0.120 \\
\hline 1 year $-<5$ years & 2538 & 44.2 & 114 & 55.1 & 0.008 \\
\hline 5 years $-<13$ years & 1187 & 20.7 & 34 & 16.4 & 0.161 \\
\hline Main diagnosis & - & & - & & $<0.001$ \\
\hline Respiratory illness & 1780 & 31.0 & 24 & 11.6 & $<0.001$ \\
\hline Diarrhoeal disease & 863 & 15.0 & 93 & 44.9 & $<0.001$ \\
\hline Other & 3095 & 53.9 & 90 & 43.5 & 0.004 \\
\hline Missing & 1 & 0.0 & 0 & 0 & - \\
\hline
\end{tabular}

The $p$-values are based on non-missing cases only.

TABLE 2: Nutritional status and Enterobacteriacae infections.

\begin{tabular}{lccccccc}
\hline Nutritional status & \multicolumn{2}{c}{$\begin{array}{c}\text { No Enterobacteriaceae } \\
\text { infection }\end{array}$} & & \multicolumn{2}{c}{$\begin{array}{c}\text { Enterobacteriaceae } \\
\text { infection }\end{array}$} & $p<0.001$ \\
\cline { 2 - 3 } & $\boldsymbol{N}$ & $\mathbf{\%}$ & & $\boldsymbol{N}$ & $\mathbf{\%}$ & \\
\hline Normal & 4672 & 82.4 & & 119 & 57.5 & $<0.001$ \\
OWFA & 244 & 4.3 & & 6 & 2.9 & 0.480 \\
UWFA & 400 & 7.1 & & 22 & 10.6 & 0.110 \\
SAM with oedema & 279 & 4.9 & & 32 & 15.5 & $<0.001$ \\
SAM without oedema & 75 & 1.3 & & 28 & 13.5 & $<0.001$ \\
Missing & 1 & 0.0 & & 0 & 0 & - \\
\hline
\end{tabular}

OWFA, overweight for age; UWFA, underweight for age; SAM, severe acute malnutrition.

The $p$-values are based on non-missing cases only.

Children classified as SAM with oedema were the group most susceptible to Enterobacteriaceae infection, and this is shown by the logistic regression analysis in Figure 2.

\section{Human immunodeficiency virus exposure}

Of the 207 Enterobacteriaceae culture-positive patients, 39 $(17.1 \%)$ were HIV-infected. Of these, 33 were on lifelong antiretroviral treatment (ART). 


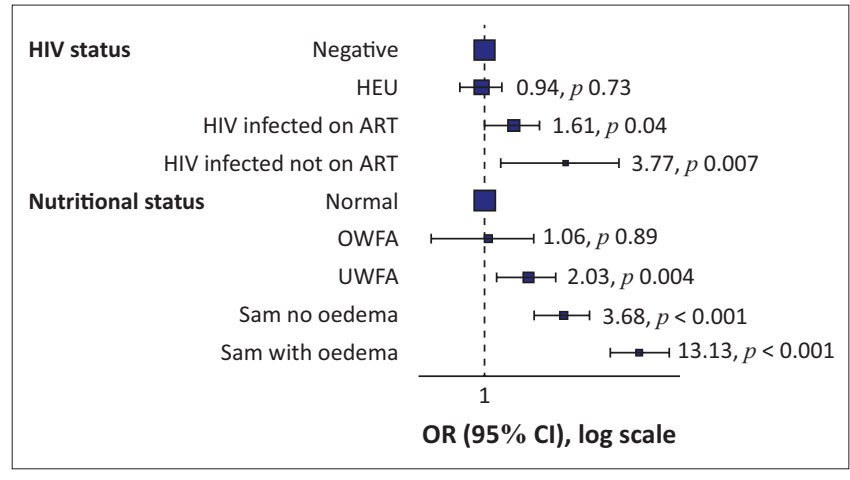

HIV, human immunodeficiency virus; HEU, HIV exposed uninfected; ART, antiretroviral treatment; OWFA, overweight for age; UWFA, underweight for age; SAM, severe acute malnutrition; $\mathrm{Cl}$, confidence interval; OR, odds ratio.

FIGURE 2: Multiple logistic regression analysis of human immunodeficiency virus status and nutrition and Enterobacteriaceae infection.

TABLE 3: Human immunodeficiency virus status and Enterobacteriacae infections.

\begin{tabular}{|c|c|c|c|c|c|}
\hline \multirow[t]{2}{*}{ HIV status } & \multicolumn{2}{|c|}{$\begin{array}{c}\text { No Enterobacteriaceae } \\
\text { infection }\end{array}$} & \multicolumn{2}{|c|}{$\begin{array}{l}\text { Enterobacteriaceae } \\
\text { infected }\end{array}$} & \multirow[t]{2}{*}{$p<0.001$} \\
\hline & $N$ & $\%$ & $N$ & $\%$ & \\
\hline Negative & 3607 & 63.6 & 112 & 54.1 & 0.010 \\
\hline $\begin{array}{l}\text { HIV-exposed } \\
\text { uninfected }\end{array}$ & 1599 & 28.2 & 55 & 26.6 & 0.637 \\
\hline Infected on cART & 380 & 6.7 & 33 & 15.9 & $<0.001$ \\
\hline Infected not on cART & 29 & 0.5 & 6 & 2.9 & 0.004 \\
\hline
\end{tabular}

HIV, human immunodeficiency virus; CART, combination antiretroviral treatment.

The $p$-values are based on non-missing cases only.

Of the HIV-infected children, a greater number of those who were not on ART had an Enterobactericiae infection(6/35; $17.1 \%)$ when compared with HIV-infected children on ART $(33 / 413 ; 8 \%)(p<0.001)$. The duration of ART, CD4 count and viral load data were not available for patients in either of these groups. Table 3 indicates the HIV status of children with and without Enterobacteriaceae infection.

\section{Comparison of nutritional status and human immunodeficiency virus infection risk to Enterobacteriaceae infections}

Figure 2 illustrates that HIV infection and malnutrition were independently associated with Enterobacteriaceae infection. Severe acute malnutrition with oedema was significantly associated with Enterobacteriaceae infections. Human immunodeficiency virus infection also posed a higher risk, less if the HIV-infected child was on ART.

\section{Duration of stay}

The mean LOS for all hospitalisations for the study period was 5.5 days, whereas $99(47 \%)$ children infected with Enterobacteriaceae stayed for longer than 7 days and more than half of these children $(54 ; 26.1 \%)$ were hospitalised for at least 2 weeks.

\section{Mortality}

Mortality was more than twice higher in children with Enterobacteriaceae infection $(10 ; 4.8 \%)$ compared with those who did not have an Enterobacteriaceae infection
$(113 ; 2.0 \%) p=0.005$. Of the children who died because of Enterobacteriaceae infection, half had a positive blood culture $(n=5)$, with the rest having non-invasive isolates (two sputum isolates, one urine isolate, one skin swab isolate, and one death had an unspecified isolate).

\section{Discussion}

This study evaluated the clinical characteristics of hospitalised children with a positive Enterobacteriaceae culture. Human immunodeficiency virus and malnutrition were significant risk factors for Enterobacteriaceae infection.

The association of HIV infection and increased risk for Enterobacteriaceae infection in children is not well understood, especially in the context of improved ART access. ${ }^{1,2}$ Human immunodeficiency virus-exposed infants may have lower body weight, an ill mother and thus may be at risk for poor growth trajectories and increased infections. Higher infection rates with Enteropathogenic E. coli (EPEC), Cryptosporidium and increased gut colonisation with ESBL Enterobacteriaceae have been found in HIV-infected children..$^{17,18,19,20}$ In our study, the incidence of Enterobacteriaceae infection was higher in children who were yet to be initiated on ART compared with those already on lifelong ART. Being ART naïve is probably associated with a compromised immune system. The independent association of HIV and malnutrition with Enterobacteriaceae infection in this study highlights the contributing role of these factors to infection risk.

In this cohort, children with SAM with or without oedema were more susceptible to Enterobacteriaceae infection. Several studies have described that patients with malnutrition are more vulnerable to Enterobacteriaceae infections. . $11,22,23,24^{2}$ Environmental enteric dysfunction (EED), villous blunting, reduction in mucus-secreting goblet cells, inflammation, reduced microbial diversity and increased potent pathogenic Enterobacteriaceae in children with malnutrition have been postulated as potential causes and warrant further study. ${ }^{25,26,27,28,29}$

In keeping with other studies, the authors found that diarrhoeal disease was strongly associated with Enterobacteriaceae infection..$^{24,30,31}$ These findings suggest that Enterobacteriaceae infection, malnutritions and diarrhoea are possibly intertwined.

The incidence of Enterobacteriaceae infection in this study (3.5\%) is similar to reports from other studies. ${ }^{15,32,33}$ The higher incidence of Enterobacteriaceae infections in children under five has also been previously reported, and the role of perineal hygiene and toilet training are considered possible contributory factors. ${ }^{34}$

Positive cultures for Enterobacteriaceae infections were predominantly from urinary samples. Several studies have demonstrated that Enterobacteriaceae organisms are the dominant Gram-negative organism causing UTIs in children. ${ }^{35,36,37}$ Urinary tract infections in children often present with non-urinary tract symptoms and are frequently 
diagnosed during investigations for sepsis, diarrhoea or malnutrition. ${ }^{38}$ The association between UTIs and malnutrition has been documented and the authors of this study confirms this association.

Enterobacteriaceae are the leading group of Gram-negative organisms causing UTIs in children, which has implications for the empiric choice of antibiotics. ${ }^{39}$ The South African Standard Treatment Guidelines recommend using amoxicillin/clavulanic acid to treat UTIs and ampicillin and gentamicin to treat children with complicated SAM or oral amoxicillin for uncomplicated SAM. ${ }^{10}$ The emergence of increasing antibiotic drug resistance, especially in hospitalised children with malnutrition, is of concern. The guidelines may need to be revised.

Increasing antibiotic resistance amongst urinary tract isolates against ampicillin, amoxicillin and cotrimoxazole has been reported. Most isolates are still sensitive to third-generation cephalosporins, quinolones, macrolides and aminoglycosides. ${ }^{40}$ Careful monitoring of antimicrobial resistance in high-risk sub-populations, such as severely malnourished children, is warranted.

Of the 207 Enterobacteriaceae cultured, 51 (24.6\%) produced extended-spectrum beta-lactamases (ESBL) in mostly community-acquired infections.

This is in keeping with other studies, where an incidence of $10 \%-35 \%$ was described. ${ }^{7,17,33}$ A recent study demonstrated the correlation between the routine use of amoxicillin in children with uncomplicated SAM and increased ESBL production. ${ }^{41}$ Amoxicillin use is recommended as one of the WHO '10 steps' in managing malnutrition. ${ }^{42}$ Children exposed to amoxicillin showed an increased ESBL production amongst isolates and a higher risk of transmission to siblings. ${ }^{41}$ Further research on the use of broad-spectrum antibiotics amongst malnourished children is required..$^{43,44,45}$

\section{Limitations}

This was a retrospective study and data were obtained from patient records, where there was no standardisation of anthropometric measurements, blood and urine sampling techniques and clinical reviews. Information on ART duration was not available, CD4 count and HIV viral load results were also not available for analysis. There were missing data, including confirmatory HIV PCR results. In this study, comparisons of infections from communityacquired and hospital-acquired and from invasive (blood, CSF) and non-invasive (urine, stool) sources could not be determined. In addition, the susceptibility of empiric choices in antibiotics could not be confirmed.

\section{Conclusion}

This study was conducted in an area with a high prevalence of HIV infection. Enterobacteriaceae infections are relatively common in hospitalised children, particularly in severely malnourished children with oedema and HIV-infected children not on ART. A high index of suspicion is necessary when caring for hospitalised children with these diagnoses. Revision of current antibiotic recommendations for hospitalised children with malnutrition may be necessary if current guidelines result in treatment failure related to antimicrobial resistance. Strategies to prevent malnutrition and HIV infection in children should be prioritised to reduce the risk of Enterobacteriaceae infections, mortality and prolonged hospital stay.

\section{Acknowledgements}

The authors would like to express their gratitude to $\mathrm{Mr}$ Partson Tinwaro for his assistance with the statistical analyses and Mrs Leora Sewnarain for assistance with formatting and language review.

\section{Competing interests}

The authors declare that they have no financial or personal relationships that may have inappropriately influenced them in writing this article.

\section{Authors' contributions}

H.K. was responsible for study design, data collection, data analysis and drafting the manuscript. K.L.N. was responsible for the supervision of the entire work, study design and manuscript review. P.M. and M.A. were responsible for manuscript review. All authors read and approved the final manuscript.

\section{Funding information}

The authors received no financial support for the research, authorship and/or publication of this article.

\section{Data availability}

The data that support the findings of this study are available from the corresponding author, H.K., upon reasonable request.

\section{Disclaimer}

The views and opinions expressed in this article are those of the authors and do not necessarily reflect the official policy or position of any affiliated agency of the authors.

\section{References}

1. United Nations International Children's Emergency Fund (UNICEF). Under-five mortality, September 2020 [homepage on the Internet]. [cited 2021 Feb 3]. Available from: https://data.unicef.org/topic/child-survival/under-five-mortality

2. World Health Organisation (WHO). Child Health, Africa. Factsheet: Children Reducing mortality [homepage on the Internet]. [cited 2021 Feb 3]. Available from: https://www.afro.who.int/health-topics/child-health\#: :text=Leading $\% 20$ causes $\% 20$ of $\% 20$ death $\% 20$ in,than $\% 20$ children $\% 20$ in $\% 20$ developed $\% 20$ regions

3. Black RE, Allen LH, Bhutta ZA, et al. Maternal and child undernutrition study group. Maternal and child undernutrition: Global and regional exposures and health consequences. Lancet. 2008;371(9608):243-260. https://doi.org/10.1016/ S0140-6736(07)61690-0

4. Aiken AM, Allegranzi B, Scott JA, et al. Antibiotic resistance needs global solutions Lancet Infect Dis. 2014;14(7):550-551. https://doi.org/10.1016/S14733099(14)70709-1

5. Dramowski A, Cotton MF, Rabie $\mathrm{H}$, et al. Trends in paediatric bloodstream infections at a South African referral hospital. BMC Pediatr. 2015;15:33. https:// doi.org/10.1186/s12887-015-0354-3 
6. Schwaber MJ, Carmeli Y. Mortality and delay in effective therapy associated with extended-spectrum beta-lactamase production in Enterobacteriaceae with extended-spectrum beta-lactamase production in Enterobacteriaceae 2007:60(5):913-920. https://doi.org/10.1093/jac/dkm318

7. Lukac PJ, Bonomo RA, Logan LK. Extended-spectrum $\beta$-lactamase-producing Enterobacteriaceae in children: Old foe, emerging threat. Clin Infect Dis. 2015;60(9):1389-1397. https://doi.org/10.1093/cid/civ020

8. Gavhi F, Kuonza L, Musekiwa A, et al. Factors associated with mortality in children under five years old hospitalised for severe acute malnutrition in Limpopo province, South Africa, 2014-2018: A cross-sectional analytic study. PLoS One. 2020;15(5):e0232838. https://doi.org/10.1371/journal.pone.0232838

9. Jones KD, Berkley JA. Severe acute malnutrition and infection. Paediatr Int Child Health. 2014;341(Suppl 1):S1-S29. https://doi.org/10.1179/204690471 $4 Z .000000000218$

10. Republic of South African Department of Health. Standard treatment guidelines and essential medicines list for South Africa [homepage on the Internet]. Hospital Level Paediatrics; 2017 [cited 2021 Feb 3]. Available from: http://www.kznhealth. gov.za/pharmacy/paediatric-stgs-eml_4thed2017.pdf

11. Nel E. Diarrhoea and malnutrition. S Afr J Clin Nutr. 2010;23(Suppl 1):15-18. https://doi.org/10.1080/16070658.2010.11734262

12. Brink A, Coetzee J, Clay C et al. The spread of carbapenem-resistant Enterobacteriaceae in South Africa: Risk factors for acquisition and prevention. S Afr Med J. 2012;102(7):599-601. https://doi.org/10.7196/SAMJ.5789

13. Logan LK. Carbapenem-resistant Enterobacteriaceae: An emerging problem in children. Clin Infect Dis. 2012;55(6):852-859. https://doi.org/10.1093/cid/cis543

14. Chiotos K, Han JH, Tamma PD. Carbapenem-resistant Enterobacteriaceae infections in children. Curr Infect Dis Rep. 2016;18(1):2. https://doi.org/10.1007/ s11908-015-0510-9

15. Flokas $M E$, Detsis $M$, Alevizakos $M$, et al. Prevalence of ESBL-producing Enterobacteriaceae in paediatric urinary tract infections: A systematic review and meta-analysis. J Infect. 2016;73(6):547-557. https://doi.org/10.1016/j. jinf.2016.07.014

16. World Health Organisation (WHO). Guideline: Assessing and managing children at primary health care facilities to prevent overweight and obesity in the context of the double burden of malnutrition: Updates for the integrated management of childhood illness (IMCl): Table 1, World Health Organization (WHO) classification of nutritional status of infants and children [homepage on the Internet]. Geneva: World Health Organization; 2017 [cited 2021 Feb 3]. Available from: https://www. ncbi.nlm.nih.gov/books/NBK487900/table/fm.s1.t1/

17. Pavlinac PB, John-Stewart GC, Naulikha JM, et al. High-risk enteric pathogens associated with HIV infection and HIV exposure in Kenyan children with acute diarrhoea. AIDS. 2014;28(15):2287-2296. https://doi.org/10.1097/ QAD.0000000000000396

18. Tellevik MG, Blomberg B, Kommedal $\varnothing$, et al. High prevalence of faecal carriage of ESBL-producing Enterobacteriaceae among children in Dar es Salaam, Tanzania. PLoS One. 2016;11(12):e0168024. https://doi.org/10.1371/journal.pone.0168024

19. Wilmore SMS, Kranzer K, Williams A, et al. Carriage of extended-spectrum betalactamase-producing Enterobacteriaceae in HIV-infected children in Zimbabwe. Med Microbiol. 2017;66(5):609-615. https://doi.org/10.1099/jmm.0.000474

20. Manyahi J, Moyo SJ, Tellevik MG, et al. High prevalence of fecal carriage of extended spectrum $\beta$-lactamase-producing Enterobacteriaceae among newly
HIV-diagnosed adults in a community setting in Tanzania. Microb Drug Resist. HIV-diagnosed adults in a community setting in Tanzania. Microb
2020;26(12):1540-1545. https://doi.org/10.1089/mdr.2020.0066

21. World Health Organisation (WHO). Interactions of nutrition and infection Scrimshaw NS, Taylor CE, Gordon JE \& World Health Organization [homepage on the Internet]. 1968 [cited 2021 Feb 3]. Available from: https://apps.who.int/iris/ the Internet]. 1968 [cite

22. Lazzerini M, Tickell D. Antibiotics in severely malnourished children: Systematic review of efficacy, safety and pharmacokinetics. Bull World Health Organ 2011;89:594-607. https://doi.org/10.2471/BLT.10.084715

23. Walson JL, Berkley JA. The impact of malnutrition on childhood infections. Curr Opin Infect Dis. 2018;31(3):231-236. https://doi.org/10.1097/QCO.000 0000000000448

24. Platts-Mills JA, Taniuchi M, Uddin MJ, et al. Association between enteropathogens and malnutrition in children aged 6-23 mo in Bangladesh A case-control study. Am J Clin Nutr. 2017;105(5):1132-1138. https://dol. org/10.3945/ajcn.116.138800

25. Attia S, Feenstra M, Swain N, et al. Starved guts: Morphologic and functional intestinal changes in malnutrition. J Pediatr Gastroenterol Nutr. 2017; 65(5):491-495. https://doi.org/10.1097/MPG.0000000000001629
26. Million M, Diallo A, Raoult D. Gut microbiota and malnutrition. Microb Pathog. 2017;106:127-138. https://doi.org/10.1016/j.micpath.2016.02.003

27. Berkley JA, Lowe BS, Mwangi I, et al. Bacteremia among children admitted to a rural hospital in Kenya. N Engl J Med. 2005;352(1):39-47. https://doi.org/10.1056/ NEJMoa040275

28. Denno DM, Tarr PI, Nataro JP. Environmental enteric dysfunction: A case definition for intervention trials. Am J Trop Med Hyg. 2017;97(6):1643-1646. https://doi. org/10.4269/ajtmh.17-0183

29. Pohanka M. Role of oxidative stress in infectious diseases: A review. Folia Microbiol. 2013;58(6):503-513. https://doi.org/10.1007/s12223-013-0239-5

30. Lima AAM, Soares AM, Filho JQS, et al. Enteroaggregative escherichia coli subclinical infection and coinfections and impaired child growth in the MAL-ED cohort study. J Pediatr Gastroenterol Nutr. 2018;66(2):325-333. https://doi. org/10.1097/MPG.0000000000001717

31. Montagnani C, Prato M, Scolfaro C, et al. Italian society of pediatric infectious diseases. Carbapenem-resistant Enterobacteriaceae infections in children: An Italian retrospective multicenter study. Pediatr Infect Dis J. 2016;35(8):862-868. https://doi.org/10.1097/INF.0000000000001188

32. Agegnehu $A$, Worku $M$, Nigussie $D$, et al. Pediatric febrile urinary tract infection caused by ESBL producing Enterobacteriaceae species. BioMed Res Int. 2020;2020:Article 6679029. https://doi.org/10.1155/2020/6679029

33. Flokas ME, Karanika $S$, Alevizakos $M$, et al. Prevalence of ESBL-producing Enterobacteriaceae in pediatric bloodstream infections: A systematic review and meta-analysis. PLoS One. 2017;12(1):e0171216. https://doi.org/10.1371/journal. pone.0171216

34. Hijazi SM, Fawzi MA, Ali FM, et al. Prevalence and characterisation of extended spectrum beta-lactamases producing Enterobacteriaceae in healthy children and associated risk factors. Ann Clin Microbiol Antimicrob. 2016;15:3. https://doi. org/10.1186/s12941-016-0121-9

35. LarcombeJ.Urinarytractinfection inchildren.BrMedJ.1999;319(7218):1173-1175. https://doi.org/10.1136/bmj.319.7218.1173

36. Shaw KN, Gorelick M, McGowan KL, et al. Prevalence of urinary tract infection in febrile young children in the emergency department. Pediatrics. 1998;102:e16. https://doi.org/10.1542/peds.102.2.e16

37. Reed RP, Wegerhoff FO. Urinary tract infection in malnourished rural African children. Ann Trop Paediatr. 1995;15(1):21-26. https://doi.org/10.1080/0272493 6.1995.11747744

38. Jeena PM, Coovadia HM, Adhikari M. Probable association between urinary tract infections (UTI) and common diseases of infancy and childhood: A hospital-based study of UTI in Durban, South Africa. J Trop Pediatr. 1996;42(2):112-114. https:// doi.org/10.1093/tropej/42.2.112

39. Brkic S, Mustafic S, Nuhbegovic S, Ljuca F, Gavran L. Clinical and epidemiology characteristics of urinary tract infections in childhood. Med Arh. 2010; 64(3):135-138.

40. Kalantar EA, Motlagh ME, Lornezhad $H$, et al. Prevalence of urinary tract pathogens and antimicrobial susceptibility patterns in children at hospitals in Iran Iran J Clin Infect Dis [serial online]. 2008 [cited 2021 Feb 3];3(3):149-153. Available from: https://www.sid.ir/FileServer/JE/122020080307

41. Maataoui N, Langendorf $C$, Berthe $F$, et al. Increased risk of acquisition and transmission of ESBL-producing Enterobacteriaceae in malnourished children exposed to amoxicillin. J Antimicrob Chemother. 2020;75(3):709-717. https://doi org/10.1093/jac/dkz487

42. World Health Organisation (WHO). Guidelines for the inpatient treatment of severely malnourished children (WHO 10 steps to managing malnutrition [homepage on the Internet]. 2003 [cited $2021 \mathrm{Feb} 3$ ]. Available from: https:// www.who.int/nutrition/publications/guide_inpatient_text.pdf

43. Page AL, De Rekeneire N, Sayadi S, et al. Infections in children admitted with complicated severe acute malnutrition in Niger. PLoS One. 2013;8(7):e68699. https://doi.org/10.1371/journal.pone.0068699

44. Lenters L, Wazny K, Bhutta ZA. Management of severe and moderate acute malnutrition in children. In: Black RE, Laxminarayan $R$, Temmerman $M$, et al. editors. Reproductive, maternal, newborn, and child health: Disease control priorities, 3rd ed. Vol. 2 [homepage on the Internet]. Washington, DC: The International Bank for Reconstruction and Development/The World Bank, 2016 [cited 2021 Feb 8]; Chapter 11. Available from: https://www.ncbi.nlm.nih.gov/ books/NBK361900/

45. Trehan I, Goldbach HS, LaGrone LN, et al. Antibiotics as part of the management of severe acute malnutrition. N Engl J Med. 2013;368(5):425-435. https://doi. org/10.1056/NEJMoa120285 